TITLE:

\title{
Resolving Nonadiabatic Dynamics of Hydrated Electrons Using Ultrafast Photoemission Anisotropy
}

$\operatorname{AUTHOR}(S)$ :

Karashima, Shutaro; Yamamoto, Yo Ichi; Suzuki, Toshinori

\section{CITATION:}

Karashima, Shutaro ... [et al]. Resolving Nonadiabatic Dynamics of Hydrated Electrons Using Ultrafast Photoemission Anisotropy. Physical Review Letters 2016, 116(13): 137601.

\section{ISSUE DATE:}

2016-04-01

URL:

http://hdl.handle.net/2433/218322

RIGHT:

(C) 2016 American Physical Society. 


\title{
Resolving Nonadiabatic Dynamics of Hydrated Electrons Using Ultrafast Photoemission Anisotropy
}

\author{
Shutaro Karashima, Yo-ichi Yamamoto, and Toshinori Suzuki* \\ Department of Chemistry, Graduate School of Science, Kyoto University, Kitashirakawa-Oiwakecho, \\ Sakyo-Ku, 606-8502 Kyoto, Japan \\ (Received 21 June 2015; revised manuscript received 30 January 2016; published 1 April 2016)
}

\begin{abstract}
We have studied ultrafast nonadiabatic dynamics of excess electrons trapped in the band gap of liquid water using time- and angle-resolved photoemission spectroscopy. Anisotropic photoemission from the first excited state was discovered, which enabled unambiguous identification of nonadiabatic transition to the ground state in $60 \mathrm{fs}$ in $\mathrm{H}_{2} \mathrm{O}$ and $100 \mathrm{fs}$ in $\mathrm{D}_{2} \mathrm{O}$. The photoelectron kinetic energy distribution exhibited a rapid spectral shift in ca. $20 \mathrm{fs}$, which is ascribed to the librational response of a hydration shell to electronic excitation. Photoemission anisotropy indicates that the electron orbital in the excited state is depolarized in less than 40 fs.
\end{abstract}

DOI: 10.1103/PhysRevLett.116.137601

A living cell exposed to high-energy particles or radiation undergoes ionization of cell water to generate $\mathrm{H}_{2} \mathrm{O}^{+}$and a quasifree electron. The former transfers a proton to a neutral water molecule, $\mathrm{H}_{2} \mathrm{O}^{+}+\mathrm{H}_{2} \mathrm{O} \rightarrow \mathrm{H}_{3} \mathrm{O}^{+}+\mathrm{OH}$, to produce an $\mathrm{OH}$ radical, which reacts with DNA to induce strand breaks. The latter undergoes dissociative attachment to molecules within its picosecond lifetime before being trapped in the band gap of liquid water. Thus, the dynamics of electrons in liquid water are of great importance in radiation chemistry and biology [1]. It is also of great interest in the study of quantum physics in soft matter [2-5].

The simplest picture of an electron trapped in liquid water [a hydrated electron; $\left.e^{-}(\mathrm{aq})\right]$ is a charged particle in a spherical dielectric cavity [6]. This widely accepted picture has been challenged [7]; however, recent calculations using density functional theory (DFT) suggest that $e^{-}$(aq) is indeed a cavity state, although the probability density of an excess electron strongly penetrates into surrounding water molecules [8-10]. The one-electron wave function of an excess electron in the ground state (GS) is nodeless like an $s$ orbital, while those of the triply degenerate excited state (ES) have a single node similar to that of $p$ orbitals. The GS is $3.3 \mathrm{eV}$ lower in energy than the vacuum level [11-14], and the ES, which mediates electron trapping from the conduction band, is ca. $1.7 \mathrm{eV}$ higher in energy than the GS. Previous experimental studies [15-18] have shown that the ES undergoes a nonadiabatic transition to the GS within 1 ps; however, neither an accurate time scale nor the mechanistic details have been established [6]. One of the difficulties faced in previous experiments [15-18] was that the ES and GS could not be resolved clearly using transient absorption and photoemission spectra. Here, we present an alternative experimental approach that allows us to resolve the nonadiabatic dynamics of $e^{-}(\mathrm{aq})$ using time- and angleresolved photoemission spectroscopy [19].

If we use an analogy with photoemission from an atom in the gas phase, we may anticipate different photoemission anisotropy for the GS and ES of $e^{-}$(aq), because the former creates a $p$ partial wave while the latter creates $s$ and $d$ waves. This, however, is too simplistic a view, because a photoelectron undergoes elastic scattering with solvent molecules prior to emission from the surface. We have shown in our previous study [19] that, despite its strong $s$ character, photoemission from the GS is isotropic, and we have attributed this to the elastic scattering of photoelectrons in bulk water. Based on this result, photoemission from the ES may also be expected to be isotropic. Nonetheless, this expectation is wrong, as we present here.

In our experiment, an aqueous $\mathrm{NaBr}$ solution was discharged into a photoelectron spectrometer from a fused silica capillary (inner diameter of $15 \mu \mathrm{m}$ ) at a flow rate of $0.18 \mathrm{~mL} / \mathrm{min}$ to create a laminar flow. Three laser pulses illuminated the laminar flow $1 \mathrm{~mm}$ downstream from the nozzle at a repetition rate of $50 \mathrm{kHz}$. The liquid temperature is estimated around $278 \mathrm{~K}$. The first pulse $\left(\omega_{1}, 200 \mathrm{~nm}\right.$, $3-10 \mathrm{~nJ})$ generated $e^{-}(\mathrm{aq})$ in the GS via a charge-transferto-solvent reaction from $\mathrm{Br}^{-}$to bulk water. The second pulse $\left(\omega_{2}, 700 \mathrm{~nm}, 240-320 \mathrm{~nJ}\right)$ optically excited $e^{-}$(aq) to the ES. The third pulse $\left(\omega_{3}, 350 \mathrm{~nm}, 60-100 \mathrm{~nJ}\right)$ induced photoemission of $e^{-}$(aq) from the ES and highly vibrationally excited states in the GS; the photon energy of $\omega_{3}$ $(3.54 \mathrm{eV})$ was only slightly larger than the vertical binding energy (VBE) of $e^{-}(\mathrm{aq})$, so that almost no photoemission was induced from the GS at equilibrium. The crosscorrelation between $\omega_{2}$ and $\omega_{3}$ pulses was estimated to be 55-80 fs, depending on the condition of the laser. The $\omega_{2}$ pulses were delayed with respect to $\omega_{1}$ by 200 ps to thermalize $e^{-}(\mathrm{aq})$ prior to the pump $\left(\omega_{2}\right)$ and probe $\left(\omega_{3}\right)$ measurements. The polarization direction of $\omega_{1}$ was perpendicular to the electron detection axis of a time-offlight (TOF) electron energy analyzer in all measurements. The polarization directions of the $\omega_{2}$ and $\omega_{3}$ pulses, indicated by $\theta_{2}$ and $\theta_{3}$ in Fig. 1(b), were varied 
(a)

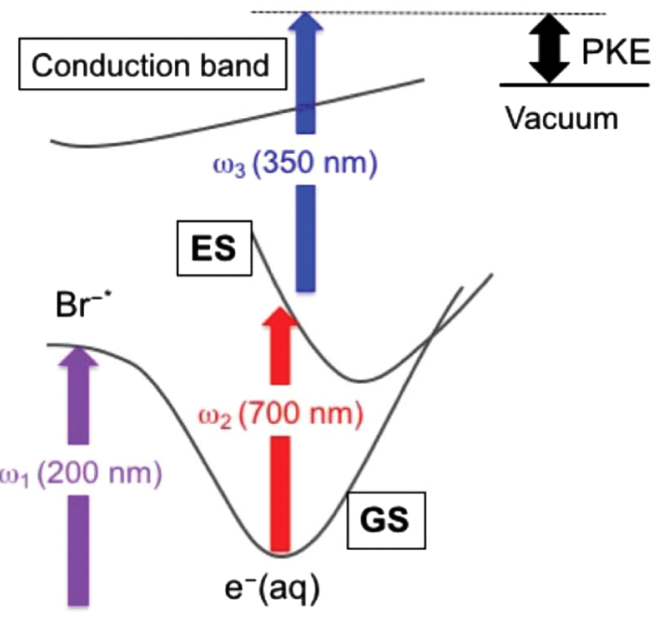

Solvation Coordinate

(b)

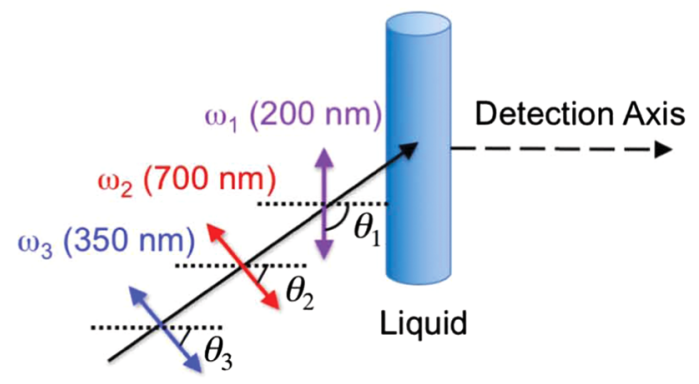

FIG. 1. (a) Schematic energy diagram and (b) experimental geometry.

independently using half wave plates. In measurements of photoemission anisotropy, the photoionization region of the apparatus was maintained field-free, and photoelectrons were sampled using a 1-mm diameter skimmer placed at $2 \mathrm{~mm}$ from the liquid surface. The TOF energy analyzer had an electron flight path of $1.2 \mathrm{~m}$, and electrostatic lenses placed in the flight tube collimated the electron trajectories and directed them to the detector. Thus, the detection solid angle $(0.18 \mathrm{sr})$ was primarily determined by the entrance skimmer. The energy resolution of the analyzer was $40 \mathrm{meV}$. The TOF spectrum was measured using a multichannel scaler. In the following discussion, the pump-probe delay time $\left(\Delta t_{23}\right)$ refers to the time interval between pulses $\omega_{2}$ and $\omega_{3}$. We also performed angleintegrated measurements by adding a SmCo permanent magnet in the ionization region and a solenoid in the flight tube to construct a magnetic bottle [20].

In order to understand the time evolution of the photoelectron kinetic energy (PKE) distribution, let us first examine the angle-integrated results. Figure 2 presents two-dimensional maps of the PKE distributions measured as a function of $\Delta t_{23}$ for $e^{-}$(aq) in (a) $\mathrm{H}_{2} \mathrm{O}$ and (b) $\mathrm{D}_{2} \mathrm{O}$; the
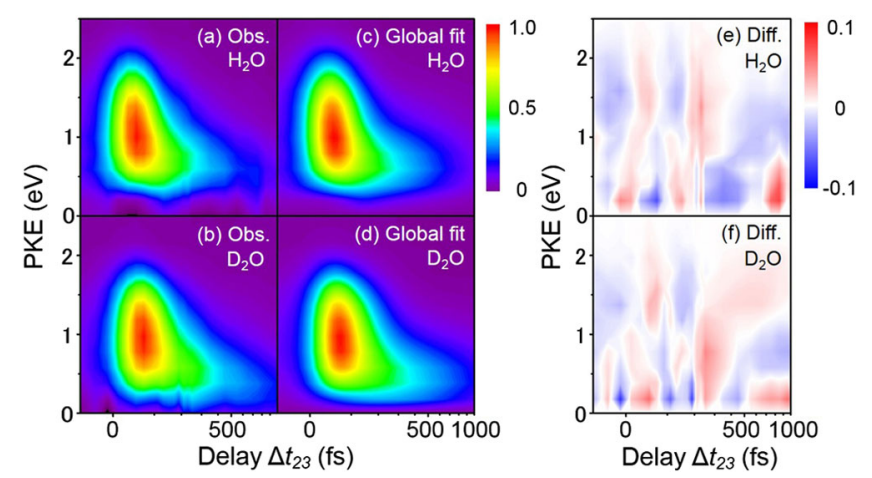

FIG. 2. Two-dimensional map of the photoelectron kinetic energy distribution measured as a function of $\Delta t_{23}$ for $e^{-}(\mathrm{aq})$ in (a) $\mathrm{H}_{2} \mathrm{O}$ and (b) $\mathrm{D}_{2} \mathrm{O}$. The global fits to these experimental data are presented in (c) and (d), and the differences between the data and the fits are shown in (e) and (f), respectively. A constant has been added to the actual delay $\left(t_{\text {plot }}=t_{\text {true }}+150 \mathrm{fs}\right)$ to shift the entire distribution and display the data around $t=0$. The time label and grids are presented for $t_{\text {true }} \cdot \theta_{2}$ and $\theta_{3}$ were $0^{\circ}$ and $90^{\circ}$, respectively. The cross-correlation time between $\omega_{2}$ and $\omega_{3}$ was $75 \mathrm{fs}$.

time axis is logarithmic. In both cases, a high PKE component (1.5-2.5 eV) appears immediately after the $\omega_{2}$ pulse and decays in about $100 \mathrm{fs}$, and a low PKE component with a long lifetime of about a picosecond is also present. The entire dynamics are slower in $\mathrm{D}_{2} \mathrm{O}$ than in $\mathrm{H}_{2} \mathrm{O}$, indicating that electron dynamics are strongly coupled with the vibrations of water molecules. Figures 2(c) and 2(d), respectively, show the global fit for the data in Figs. 2(a) and 2(b) using the following function,

$$
\begin{aligned}
& f(t)=\left\{\sum_{j=1}^{N} C_{j} F(t)+B \exp \left[t / \tau_{-1}\right]\right\} \otimes g(t), \\
& F(t)= \begin{cases}0 & \left(t<t_{d}\right) \\
\exp \left[-\left(t-t_{d}\right) / \tau_{j}\right] & \left(t_{d} \leq t\right)\end{cases}
\end{aligned}
$$

where $t$ is $\Delta t_{23}, C$ and $B$ are expansion coefficients, and $\tau_{j}$ and $\tau_{-1}$ are the time constants in the positive and negative time range, respectively. $g(t)$ is a Gaussian-shaped crosscorrelation function between $\omega_{2}$ and $\omega_{3}$. The signal in the negative time region is due to a small background caused by the $\omega_{3}-\omega_{2}$ pulse sequence. Careful examination of Fig. 2(a) reveals that the peak of the PKE distribution rapidly downshifts within 20 fs due to the vibrational wave packet dynamics of the hydration shell. To express this fast evolution, we introduced the term $t_{d}$ into Eq. (1), which will be discussed in more detail later. The observed time profiles were well reproduced using Eq. (1) with $N=2$ and the following best-fit parameters: $\tau_{1}=60 \pm 10, \tau_{2}=520 \pm$ 30 , and $\tau_{-1}=180 \pm 60$ fs for $\mathrm{H}_{2} \mathrm{O}$ and $\tau_{1}=100 \pm 20$, $\tau_{2}=740 \pm 130$, and $\tau_{-1}=100 \pm 20$ fs for $\mathrm{D}_{2} \mathrm{O}$. The $\tau_{1}$ and $\tau_{2}$ time constants obtained from our analysis are in 


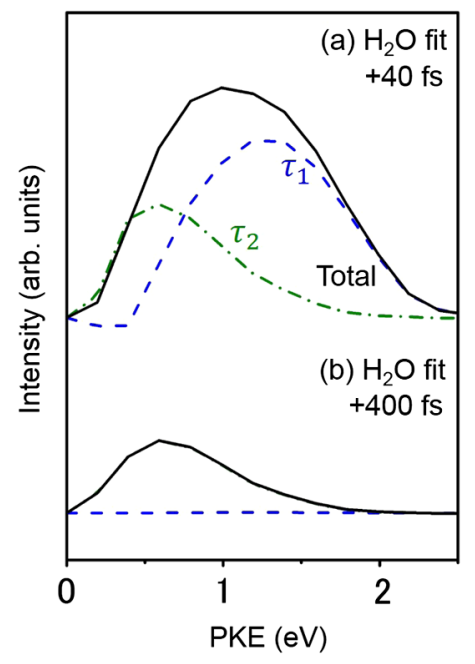

FIG. 3. Decay-associated spectra of $\tau_{1}$ (blue, dashed line) and $\tau_{2}$ (green, dashed-dot line) components and their sum (black, solid line) at $\Delta t_{23}$ values of (a) 40 and (b) 400 fs determined by global fitting using Eq. (1) with $N=2$ for $e^{-}(\mathrm{aq})$ in $\mathrm{H}_{2} \mathrm{O}$.

reasonable agreement with previous reports [15-18]. The difference between the experimental data and the global fits are shown in Figs. 2(e) and 2(f) for $\mathrm{H}_{2} \mathrm{O}$ and $\mathrm{D}_{2} \mathrm{O}$, respectively.

Figure 3 shows decay-associated spectra at $\Delta t_{23}=40$ and $400 \mathrm{fs}$ obtained by the global fit shown in Fig. 2. The $\tau_{1}$ component observed in the high PKE region is readily assigned to ES, as it appears immediately after the pump pulse, while the $\tau_{2}$ component at low PKE can be assigned to either the vibrationally relaxed ES or the highly vibrationally excited GS. The assignment of the $\tau_{2}$ component is the key question to address in this study.

Figure 4 presents time- and angle-resolved photoemission spectra of $e^{-}$(aq) in $\mathrm{H}_{2} \mathrm{O}$ at (a) $\Delta t_{23}=0$, (b) 40 , (c) 80 , and (d) $400 \mathrm{fs}$. Upper and lower panels show the results obtained using $\theta_{2}$ being $0^{\circ}$ and $90^{\circ}$, and each of eight figures shows three PKE distributions measured using $\theta_{3}$ being $0^{\circ}$ (black), $54^{\circ}$ (green), and $90^{\circ}$ (blue). The photoemission intensity at short time delay is stronger for $\theta_{3}=0^{\circ}$ than $90^{\circ}$, indicating that photoemission preferentially occurs along the electric field direction of the probe pulse. This accords with classical electrodynamics, which is seen most typically in photoemission. The $\theta_{3}$ dependence vanishes at $400 \mathrm{fs}$, when the $\tau_{2}$ component dominates. Figure 4 clearly shows that the photoemission anisotropy is associated with the $\tau_{1}$ component (ES).

The observed distributions can be simulated using the decay-associated spectra in Fig. 3 and anisotropy parameters assumed for the $\tau_{1}$ and $\tau_{2}$ components. The angular dependence, $I\left(\theta_{3}\right)$, upon two-photon photoemission is generally expressed as

$$
I\left(\theta_{3}\right) \propto 1+\beta_{2} P_{2}\left(\cos \theta_{3}\right)+\beta_{4} P_{4}\left(\cos \theta_{3}\right),
$$

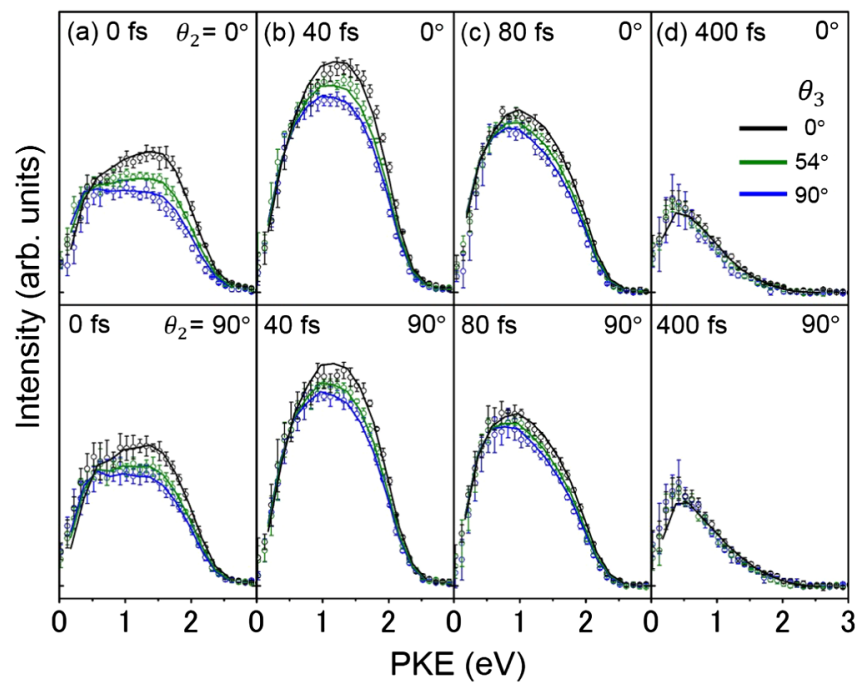

FIG. 4. Photoelectron kinetic energy distributions observed for $e^{-}$(aq) in $\mathrm{H}_{2} \mathrm{O}$ for $\Delta t_{23}=0$ values of (a) 0 , (b) +40 , (c) +80 , and (d) +400 fs. $\theta_{2}$ is $0^{\circ}$ and $90^{\circ}$ for the upper and lower panels, respectively. The black, green, and blue colors correspond, respectively, to $\theta_{3}$ of $0^{\circ}, 54^{\circ}$, and $90^{\circ}$. The dots and error bars represent the experimental data and their standard deviation, while the solid lines are simulations using the decay associated spectra and the anisotropy parameter $\left(\beta_{2}\right)$ assumed for the $\tau_{1}$ component. The anisotropy parameter of the $\tau_{2}$ component was assumed to be zero. The cross-correlation time between $\omega_{2}$ and $\omega_{3}$ was 55 fs.

where $E$ is the PKE, $\beta_{n}$ is an expansion coefficient, and $P_{n}$ is the $n$th order Legendre polynomial. In our analysis, we have neglected the $\beta_{4}$ term, which is usually small. The solid lines in both the upper and lower panels of Figs. 4(b)-4(d) are distributions simulated by assuming $\beta_{2}=0.16$ and 0 , respectively, for the $\tau_{1}$ and $\tau_{2}$ components, which are in excellent agreement with the experimental data. The difference in photoemission anisotropy between the $\tau_{1}$ and $\tau_{2}$ components suggests that they correspond to different electronic states. Since the $\tau_{1}$ component corresponds to the ES and photoemission from the GS is known to be isotropic, the $\tau_{2}$ component is assigned to a highly vibrationally excited state in the GS. Thus, we conclude that $e^{-}(\mathrm{aq})$ in $\mathrm{H}_{2} \mathrm{O}$ undergoes a nonadiabatic transition from the ES to the GS in $60 \pm 10$ fs.

Why does the ES exhibit photoemission anisotropy, even if the GS does not? We conjecture that it originates from a larger electron density for the ES at the top molecular layer of the liquid. Uhlig et al.have calculated the spin density in the GS and ES of $e^{-}$(aq) near the water surface [21], and they showed that the spin density in the inner cavity is $40 \%$ in the GS while it is less than 7\% in the ES. In contrast, in the gas phase, the fraction is $11 \%$ in the GS, while it is $20 \%-27 \%$ in the ES [21]. Although these fractions will strongly fluctuate with thermal motion of the hydrogenbonding network in liquid water, a more spread-out electron distribution in the ES than in the GS is always 
predicted for a particle in an attractive potential with a finite barrier. The $\beta_{2}$ value of 0.16 is relatively low, because photoemission from $e^{-}(\mathrm{aq})$ at larger depths becomes isotropic. The probing depth of photoemission spectroscopy is still under investigation [22-24]; however, it is on the order of nanometers in the PKE region of $5 \mathrm{eV}$, which is greater than the radius of gyration of $e^{-}(\mathrm{aq})(0.25 \mathrm{~nm})$.

The linearly polarized $\omega_{2}$ pulses create orbital alignment of the ES. It is anticipated that the $p$ orbital alignment perpendicular to the liquid surface is more effective than the parallel alignment to increase the excess electron density at the top molecular layer and to enhance photoemission anisotropy. Therefore, we initially employed $\theta_{2}=0$ (the upper panels in Fig. 4). Then, we performed similar measurements for $\theta_{2}=90$ (the lower panels in Fig. 4), which prepares the $p$ orbital parallel to the liquid surface all around the cylindrical liquid microjet. As seen in Fig. 4(a), the distribution at $t=0$ fs exhibits higher anisotropy for $\theta_{2}=0$ than for 90 . More specifically, the $\beta_{2}$ values of the $\tau_{1}$ component were 0.32 and 0.24 for $\theta_{2}=0$ and 90 , respectively. However, the distributions observed after the time delay of $40 \mathrm{fs}$ [Figs. 4(b)-4(d)] were independent of $\theta_{2}$ and expressed using the anisotropy parameters $\beta_{2}=0.16$ and 0 for the $\tau_{1}$ and $\tau_{2}$ components, respectively. The results indicate that the orbital alignment of the ES influences the photoemission angular anisotropy, and that the orbital is depolarized in less than $40 \mathrm{fs}$.

Close examination of Fig. 2 reveals that the peak energy of the PKE distribution downshifts within 20 fs. In our analysis, we expressed the spectral shift by introducing $t_{d}$ in Eq. (1). The $t_{d}$ values determined using the global fit $(N=2)$ are shown in Fig. 5. The value of $t_{d}$ is nearly zero at the highest PKE and increases for lower PKE, indicating that the highest PKE signal is from the Franck-Condon

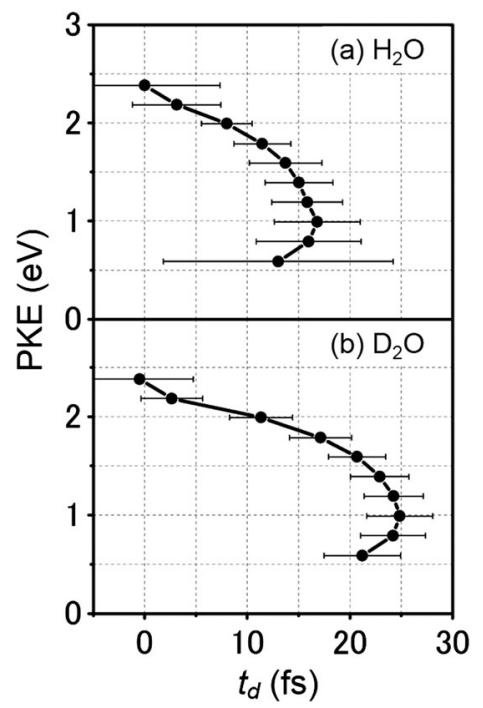

FIG. 5. Values of $t_{d}$ determined by least-squares fitting using Eq. (1) for a hydrated electron in (a) $\mathrm{H}_{2} \mathrm{O}$ and (b) $\mathrm{D}_{2} \mathrm{O}$. region in the ES, and the delayed appearance of the low PKE signal is due to wave packet motion in the ES. The downshift in the PKE indicates that the potential gradient along the reaction coordinate is greater in the ES than the final state of neutral water, so that the VBE of the ES increases along this coordinate. The $t_{d}$ value is systematically greater for $\mathrm{D}_{2} \mathrm{O}$; at a $\mathrm{PKE}$ of $1 \mathrm{eV}$, the maximum value of $t_{d}$ is ca. $17 \mathrm{fs}$ in $\mathrm{H}_{2} \mathrm{O}$, while it is $25 \mathrm{fs}$ in $\mathrm{D}_{2} \mathrm{O}$. The isotope effect suggests that the ultrafast wave packet motion responsible for the PKE shift is primarily libration of water molecules [5]. The photoelectron total intensity does not exhibit 10-20 fs time constants, so that there is negligible population decay due to the ES-GS nonadiabatic transitions within this ultrafast solvent response time. As we have seen in Fig. 4, photoemission anisotropy diminishes within the same time scale.

In conclusion, photoexcited $e^{-}$(aq) in water undergoes ultrafast solvent response of a hydration shell and depolarization of the $p$ orbital followed by a nonadiabatic transition to the GS in sub-100 fs. The subsequent vibrational relaxation from highly vibrationally excited states in the GS occurs in a subpicosecond time scale. This study confirms that the ES-GS nonadiabatic reaction time is shorter than the theoretical estimates [6] reported so far.

This work was supported by JSPS KAKENHI Grant No. 15H05753. Y. I. Y. is supported by the Research Fellowship of Japan Society for the Promotion of Science for Young Scientists. We thank M. Hayashi for contribution in the early stage of this work and S. Adachi for technical assistance.

*suzuki@kuchem.kyoto-u.ac.jp

[1] E. Alizadeh and L. Sanche, Chem. Rev. 112, 5578 (2012).

[2] A. Migus, Y. Gauduel, J. L. Martin, and A. Antonetti, Phys. Rev. Lett. 58, 1559 (1987).

[3] F. J. Webster, J. Schnitker, M. S. Friedrichs, R. A. Friesner, and P. J. Rossky, Phys. Rev. Lett. 66, 3172 (1991).

[4] C. Silva, P. K. Walhout, K. Yokoyama, and P. F. Barbara, Phys. Rev. Lett. 80, 1086 (1998).

[5] M. F. Emde, A. Bultuska, A. Kummrow, M. S. Pshenichnikov, and D. A. Wiersma, Phys. Rev. Lett. 80, 4645 (1998).

[6] L. Turi and P. J. Rossky, Chem. Rev. 112, 5641 (2012).

[7] R. E. Larsen, W. J. Glover, and B. J. Schwartz, Science 329, 65 (2010).

[8] I. A. Shkrob, J. Phys. Chem. A 111, 5223 (2007).

[9] F. Uhlig, O. Marsalek, and P. Jungwirth, J. Phys. Chem. Lett. 3, 3071 (2012).

[10] A. Kumar, J. A. Walker, D. M. Bartels, and M. D. Sevilla, J. Phys. Chem. A 119, 9148 (2015).

[11] K. R. Siefermann, Y. Liu, E. Lugovoy, O. Link, M. Faubel, U. Buck, B. Winter, and B. Abel, Nat. Chem. 2, 274 (2010).

[12] Y. Tang, H. Shen, K. Sekiguchi, N. Kurahashi, T. Mizuno, Y. I. Suzuki, and T. Suzuki, Phys. Chem. Chem. Phys. 12, 3653 (2010). 
[13] A. Lübcke, F. Buchner, N. Heine, I. V. Hertel, and T. Schultz, Phys. Chem. Chem. Phys. 12, 14629 (2010).

[14] A. T. Shreve, T. A. Yen, and D. M. Neumark, Chem. Phys. Lett. 493, 216 (2010).

[15] K. Yokoyama, C. Silva, D. H. Son, P. K. Walhout, and P. F. Barbara, J. Phys. Chem. A 102, 6957 (1998).

[16] M. Assel, R. Laenen, and A. Laubereau, J. Phys. Chem. A 102, 2256 (1998).

[17] M.S. Pshenichnikov, A. Baltuska, and D. A. Wiersma, Chem. Phys. Lett. 389, 171 (2004).

[18] M. H. Elkins, H. L. Williams, A. T. Shreve, and D. M. Neumark, Science 342, 1496 (2013).
[19] Y. I. Yamamoto, Y. I. Suzuki, G. Tomasello, T. Horio, S. Karashima, R. Mitric, and T. Suzuki, Phys. Rev. Lett. 112, 187603 (2014).

[20] P. Kruit and F. H. Read, J. Phys. E: Sci. Instrum. 16, 313 (1983).

[21] F. Uhlig, O. Marsalek, and P. Jungwirth, J. Phys. Chem. Lett. 4, 338 (2013).

[22] S. Thürmer, R. Seidel, M. Faubel, W. Eberhardt, J. C. Hemminger, S. E. Bradforth, and B. Winter, Phys. Rev. Lett. 111, 173005 (2013).

[23] Y. I. Suzuki, K. Nishizawa, N. Kurahashi, and T. Suzuki, Phys. Rev. E 90, 010302 (2014).

[24] F. Buchner, T. Schultz, and A. Lübcke, Phys. Chem. Chem. Phys. 14, 5837 (2012). 